\title{
Entre lo orgánico y lo inorgánico: la experiencia del hacerse cosa en Perniola y Braidotti
}

Antonio Pomposini

Pontificia Universidad Católica del Perú

Resumen: El presente artículo tiene como finalidad explorar los trabajos de dos autores contemporáneos: Mario Perniola y Rosi Braidotti. Ambos defienden un materialismo inmanente en el que encuentran que no puede haber una distinción radical entre el hombre y la materia que le rodea. No se puede ya apelar a un ideal trascendental de la razón si se quiere entender y describir la sociedad contemporánea. Cada autor da cuenta, desde su postura, de cómo se ha transformado la noción de subjetividad, cuál es la importancia de lo diferente y qué rol debe cumplir la sensibilidad, explorando así las formas en las que el hombre confluye con el mundo de lo inorgánico en este contexto posthumano. Este artículo expone la postura de ambos autores para luego explorar en qué medida hay posibles vínculos y relaciones y cuáles son sus principales diferencias.

Palabras clave: Perniola, Braidotti, inorgánico, materialismo, posthumanismo

Abstract: The objective of this article is to explore the work of two contemporary authors: Mario Perniola and Rosi Braidotti. They both defend an immanent materialism in which they find that there can be no radical distinction between human beings and the matter that surrounds them. An appeal to transcendental reason is no longer possible for them if one wants to understand and describe contemporary society. Each author explains, from their viewpoint, how the notion of subjectivity has been transformed, what the importance of difference is and what the role of sensibility should be, exploring, thusly, the ways in which the human interacts and melds with the world of the inorganic in this posthuman context. This article presents the positions of both authors in order to explore the extent to which possible connections and agreements can be drawn, as well as their key differences.

Key words: Perniola, Braidotti, inorganic, materialism, posthumanism 


\section{§1. Introducción}

Hoy en día estamos presenciando cambios y transformaciones drásticos en nuestra sociedad, formas de vida y concepciones del mundo. En el centro de todas ellas se encuentra la entrada del hombre en el mundo de la cosa y la disolución de la distinción entre lo animado y lo inanimado. Los recientes desarrollos científicos en robótica, informática y biogenética no hacen más que poner en evidencia la realidad a la que nos enfrentamos. La figura del cyborg se vuelve el símbolo de esta convergencia entre lo natural y lo artificial, lo orgánico y lo inorgánico, la confluencia absoluta entre el humano y la máquina. La forma en que entendemos a la subjetividad y cómo el sujeto interactúa con el mundo debe, correspondientemente, adecuarse a esta nueva realidad y abandonar presupuestos antiguos $u$ obsoletos que corresponden a una época distinta.

En este artículo, me propongo explicar y comparar los intentos de comprender la realidad actual que ofrecen dos filósofos: Mario Perniola y Rosi Braidotti. Ambos toman una postura materialista inmanente que critica la metafísica tradicional y los dualismos de esta, buscando entender la complejidad de la realidad misma. En primer lugar, exploraré dos problemas que identifica Perniola, el de la comunicación y el del extrañamiento del sentir, para de ahí mostrar de qué forma aprovecha la característica del "hacerse cosa" del hombre para hacer frente a estos. Luego expondré la tesis de Braidotti sobre la necesidad de pensar una relación sostenible con el ambiente y de repensar nuestros supuestos humanistas para afrontar la crisis ecológica capitalista que atravesamos. Finalmente, haré una reflexión respecto de ambas posturas y discutiré algunas posibles relaciones y conflictos.

\section{§2. La problemática contemporánea y la cosa que siente en Perniola}

Mario Perniola es un autor profundamente preocupado por pensar la condición contemporánea y permanecer en contacto con la realidad y con el momento presente. Esta vocación lleva a su pensamiento a realizar 
distinciones importantes que ayuden a dar cuenta, de forma acertada, de la realidad. Para este propósito, Perniola encuentra en el enigma el concepto propicio para interpretar el momento presente. Tanto los conflictos sociales como las posibles salidas deberán asumir la figura del enigma si pretenden ser efectivos, pues esta da cuenta propiamente de la realidad. Por ello, una distinción central que debemos señalar es la que nuestro autor hace con respecto al enigma y el secreto.

No es el secreto, sino el enigma, la noción que permite explicar, según Perniola, la sociedad del espectáculo integrado, como la describe Debord. En ella, por ejemplo, los gobernantes (y las demás personas, por supuesto) pueden no saber lo que está ocurriendo. El secreto, por el contrario, trae consigo una noción simplista de la verdad. Esto es, una verdad que permanece oculta, pero que está a la espera de que alguien la encuentre y la revele para que todo quede claro. Dentro de esta noción aún prevalece la idea de que la verdad es algo que se puede encontrar, señalar y fijar. En el enigma, en cambio, el misterio es algo permanente, no una condición que se disuelve a partir de la revelación de un secreto; es algo que nos deja perplejos aún frente al intento de explicarlo. La noción de verdad que sugiere el enigma es una mucho más compleja que la del secreto, ya que esta no se agota en las distintas interpretaciones que pueden hacerse de un enigma; todas ellas pueden ser sensatas y coherentes, pero ninguna zanja la cuestión de forma definitiva. Mientras que el secreto es poseído por un pequeño grupo de personas (digamos, la élite gobernante o con poder económico), el enigma surge cuando el secreto se les escapa de las manos al enfrentarse a una realidad mucho más compleja de la que esperaban. Por ejemplo:

(...) en el marco de la sociedad del espectáculo integrado, delineada por Debord, es lo que ocurre cuando los gobernantes se encuentran en el punto en el que confluyen innumerables tramas que en su mayor parte se les escapan: una sociedad en la que nadie sabe ya qué es lo que realmente sucede, en la que resulta imposible calcular exactamente el precio de la producción de quien sea, en la que la incertidumbre está organizada en todos los ámbitos (Perniola 2006b, 23).

Esta situación enigmática, en la que nadie sabe lo que está pasando, es la que Perniola describe en Contra la comunicación. Ahí, siguiendo a Umberto 
Eco, Perniola trae a colación el concepto de "semiosis hermética" como una forma peculiar de esoterismo. El esoterismo tiene un claro vínculo con la figura del secreto, puesto que aquel se preocupa por resguardar una serie de conocimientos y protegerlos de la divulgación y su consiguiente banalización. En cambio, la semiosis hermética ya no tiene un núcleo de conocimientos que protege; "[partiendo] de la premisa de que todas las ideas contienen una semilla de verdad, aunque se contradigan, la semiosis hermética establece una relación de analogía, continuidad y semejanza entre todas las cosas del universo. De este modo, cualquier afirmación resulta inadecuada y requiere que la complementen todas las otras: dado que estas últimas son infinitas, se priva así al lenguaje de la posibilidad de decir algo sensato" (Perniola 2006a, 19-20). La semiosis hermética ya no guarda relación con una verdad que puede descubrirse para darle sentido a lo que pasa. Por el contrario, hay una incertidumbre generalizada en la que diversas opiniones, incluso contradictorias, se sostienen en el mismo fenómeno. Por ello es que este fenómeno se entiende mejor a partir de la figura del enigma.

Ahora bien, la semiosis hermética resulta relevante para entender la problemática de la comunicación de masas, a la que Perniola refiere llanamente como comunicación. Esta es una forma de oscurantismo populista caracterizada por "[eludir] toda determinación como si de la peste se tratara. [Aspirar] a ser a un tiempo una cosa, su opuesto y todo aquello que está en el medio entre ambos extremos" (Perniola 2006a, 18). La comunicación presenta dos características que son centrales y merecen ser discutidas, el vitalismo y la psicosis. Por vitalismo, Perniola entiende:

[La idea] de derribar toda la lógica y racionalidad en el nombre de la inmediatez, de la espontaneidad, de la creación desde cero. La percepción de los opuestos es considerada como una jaula de la cual es preciso liberarse, una prisión que impide la libre expresión del espíritu creativo, un obstáculo que hay que abatir con un empellón: de ello se sigue la exaltación de la fuerza y la irrisión hacia aquello que aparece como meramente formal, mecánico, repetitivo, mediato, de progresión lenta, ritual, o siquiera dotado de una identidad precisa. La vida es, en realidad, esto y aquello, sin exclusiones ni impedimentos: imprevisible, contradictoria, irracional, desborda todas las barreras y no tiene que justificar ante nadie su tumultuoso curso (Perniola 2006a, 34-35). 
En otras palabras, el vitalismo toma a la vida como un valor, incluso como el sumo valor, y prioriza la expresión de la creatividad y de la fuerza vital por encima de las mediaciones que buscan instaurar un filtro de rigor o calidad sobre qué vale la pena. Para la comunicación todo vale, y vale igual. De esta forma se acepta cualquier tontería junto a las expresiones de ingenio y se termina por destruir cualquier criterio de valor o diferenciación.

La segunda característica, la psicosis, se entiende con relación a la neurosis. Mientras que en la neurosis hay un contenido reprimido que debe regresar a la conciencia, la psicosis excluye el orden simbólico de entrada, de forma que no es que "esté oculto", sino que no está ahí en primer lugar; ha sido forcluido ${ }^{1}$ (Perniola 2006a, 45-46). Este orden simbólico es entendido como la mediación del lenguaje que permite representar la realidad poniendo símbolos que configuran sentido, pero que, al hacerlo, introduce también una ruptura con la inmediatez de la experiencia primordial, presemántica. La entrada al orden simbólico supone, simultáneamente, una ventana de acceso a la alteridad y la diferencia ya que se entra al ámbito de la interpretación y posibilita, además, el malentendido y el error. Uno no puede forzar que la realidad sea entendida como él quiere que se entienda, sino que debe aceptar que otros le digan que no lo ven así.

El psicótico, al excluir el orden simbólico, es incapaz de percibir al otro y tolerar una perspectiva diferente a la suya. En cambio, quedándose en la dimensión de lo imaginario², solo tiene una proyección de sí mismo, una fantasía a la que se enfrenta (y de la que es parte), y no a una alteridad real. El individuo psicótico es prisionero "del fenómeno psicológico que Lacan definió como estadio del espejo. Este estadio se refiere a la confrontación que el niño realiza, entre los seis y dieciocho meses de vida, entre la imagen

1 Término lacaniano que refiere a una expulsión o rechazo radical; "a diferencia de lo que ocurre en la represión, [lo forcluido] es algo que no puede retornar a la conciencia del psicótico, porque nunca estuvo, fue descartado, por así decir, ab origine" (Perniola 2006a, 45-46).

2 Este concepto, junto con el de lo simbólico y el estadio del espejo, son términos lacanianos complejos. En el presente artículo no busco entrar en la complejidad de estos términos, sino simplemente hacer referencia a ellos en tanto Perniola los utiliza para su explicación de la comunicación. Él lo entiende como la proyección de la propia imagen, caracterizada "precisamente por un narcisismo inseparable de la agresividad en relación con el otro" (Perniola 2006a, 49). 
de sí mismo que ve reflejada en el espejo y la experiencia del propio cuerpo real: mientras este último carece aún de coordinación y de unidad, la imagen especular tiene sobre él un poder de fascinación y de seducción que lo apresa en una fijación invalidante" (Perniola 2006a, 48). El individuo que queda atrapado en el estadio del espejo se identifica con su imagen especular, que es una versión idealizada e ilusoria del yo. Esta identificación es compacta, es decir, el individuo que se identifica con su imagen especular cree que esta da cuenta de quién es él y agota su identidad (no puede ir más allá, o creer que también puede ser de otra forma). Así, el individuo psicótico es incapaz de concebir la posibilidad de que la realidad sea de una forma distinta a lo que su delirio le indica. Por ello, en la comunicación, como en el individuo psicótico, no hay una experiencia real o efectiva del conflicto, ya que se carece de la experiencia del opuesto, del otro, frente al cual uno está en conflicto. "De hecho, la comunicación, cuando adopta la forma de un debate público, no es una arena donde dos contendientes se enfrentan, y menos aún una disputa entre dos maestros de la universidad medieval. Se basa en un presupuesto tácito y universalmente aceptado: la exigencia dirigida al público de que se identifique con uno u otro de los antagonistas" (Perniola 2006a, 50).

La psicosis comunicativa no solo resalta la incapacidad de encontrar sentido en un discurso indescifrable, cuya clave de interpretación ha sido excluida junto con toda mediación del orden simbólico, sino que es, además, agresiva y violenta ${ }^{3}$. La exhortación a identificarse con una postura genera una actitud maniquea y simplista que ve en lo diferente un enemigo que debe ser aniquilado para afirmarse como lo único que hay. La experiencia real del conflicto no supone la aniquilación del oponente, ni mucho menos una exclusión que evite la entrada de este al conflicto, poniendo en su lugar una fantasía, sino una genuina consideración del otro, una actitud de "tomárselo en serio" y no buscar reducirlo de forma simplista o maniquea. Dada la capacidad de considerar al oponente en toda su seriedad, podemos entender que la respuesta no sea agresiva, sino moderada; que se fomente la capacidad de escuchar al otro y reconocer valor en lo que dice, sin que

3 Esto se debe a la incapacidad del psicótico para tolerar una perspectiva diferente, de aceptar que hay una falta y que no es omnipotente. 
por ello se deba abandonar la propia postura y se deje de discrepar. Aquello puede dar paso a que uno encuentre en los argumentos del oponente un verdadero desafío al que debe responder si busca tomarse su propia postura seriamente $y$, en caso no poder responder, dar cuenta de un problema real en dicha postura. No se trata de vencer a toda costa, de identificarse primero con una posición y usar toda táctica disponible para desbaratar al oponente. No hay, por ello, una pulsión desmedida de afirmarse como el único discurso existente, fijar la única verdad, sino un detenimiento y una escucha reales.

Sin embargo, aunque pensar el conflicto haya sido siempre la tarea de la filosofía, no es sino con Nietzsche, y posteriormente con Heidegger, que la oposición se entiende de manera más radical. Nietzsche consideraría cómo la tradición metafísica de occidente ha sido incapaz de concebir realmente a lo opuesto, al concebirlo en función de lo idéntico (como un idéntico que se opone a otro) (Perniola 2006a, 53). Lo otro no debería pensarse como otra identidad, sino como algo radicalmente distinto. Heidegger, por su parte, introduce la noción de diferencia y piensa lo radicalmente diferente como "algo para lo cual no valen los principios de identidad, de no contradicción y de tercero excluido (...), algo que se op-pone al pensamiento, a la voluntad y al sentimiento humanos de una manera tan radical que no puede experimentarse ni siquiera como un opuesto [aristotélico]" (Perniola 2006a, 57). Conviene subrayar, como lo hace Perniola (2006a, 59), que el pensamiento de la diferencia no supone la disolución de toda identidad en una unidad mística, sino que comprende el ímpetu de pensar lo otro de forma radical y seria.

En Enigmas, Perniola señala que lo opuesto al enigma es lo banal. El enigma tiene que ver con el tránsito de lo mismo a lo mismo, con la diferencia y el momento histórico. Lo banal, más bien, tiene que ver con "la permanencia de la identidad en sí misma, la afirmación de la categoría tautológica a través de la cual una cosa se da ni más ni menos que por sí misma. Es evidente que la sociedad del espectáculo integrado es el lugar por excelencia de la banalidad por los procesos de reducción, de homologación, de nivelación que ésta implica" (Perniola 2006b, 24). El intento de fijar la realidad, que Nietzsche identifica con la metafísica occidental, es una cara de la moneda, 
cuya inversa es la comunicación, la abolición de todo sentido. Aquí, Perniola identifica al fundamentalismo religioso como forma por excelencia de esa banalidad, ya que pretende fijar principios universales y eternos, negando así una conciencia histórica y pretendiendo afirmarse como la única verdad. Pero ese ímpetu de fijar, que está íntimamente vinculado a la relación dual entre secreto y revelación, se supera en el enigma, pues le son ajenas las concepciones dualistas y simplistas.

El enigma no está solo en el hombre o solo fuera de él, sino que pensamiento y realidad están unidos por una pertenencia mutua esencial. Es en la experiencia del enigma donde los hombres encuentran la garantía de la comunicación entre ellos, la certeza de pertenecer a un único mundo común a todos: quien, en cambio, está encerrado en la identidad, en la particularidad, en la fijeza del mundo propio, es comparado por Heráclito con un durmiente al que le falta relación con lo real (Perniola 2006b, 30).

Es importante enfatizar que el reconocimiento del tránsito en el enigma va acompañado de un reconocimiento del otro y del conflicto. Aquel que se queda en la fijeza del mundo propio no tendría, entonces, contacto con la realidad, que es ella misma enigmática. De la misma forma, el psicótico, que excluye la mediación del orden simbólico y se queda en su mundo particular, incomunicable, no tiene contacto con la realidad; excluye la experiencia del enigma y con ella, la pertenencia a un mundo común.

Ahora bien, aunque quede en claro la importancia de pensar la diferencia y asumir el enigma, antes de entrar a discutir cómo considera Perniola que esto se llega a dar, es importante detallar una característica más que Perniola identifica en la sociedad contemporánea. Esta es el especularismo y, detrás de este, la experiencia del hombre de volverse cosa. Tanto en Enigmas como en Del sentir, Perniola señala la importancia que se le ha dado en nuestra época a la imagen y al narcisismo; sin embargo, aunque reconoce valor en este diagnóstico, considera que no es del todo acertado. Perniola distingue al especularismo del narcisismo en lo siguiente: "Si para el narcisista el mundo es un espejo en el que se mira a sí mismo, la experiencia de lo ya sentido es como volverse el espejo donde se mira el mundo. Por ello, más que narcisismo, quizá habría que hablar de una manera especular que refleja experiencias ya prefiguradas" (Perniola 2008, 36). Perniola sostiene que vivimos en un momento donde ya no hay una experiencia subjetiva, 
personal e íntima, sino que estamos presenciando un extrañamiento de nuestra capacidad de sentir. Esto lo notamos al compartir una experiencia que hayamos tenido en redes sociales y solo saber cómo nos sentimos a partir de la reacción que nuestro relato suscite. Así como la ideología configuraba un ya pensado que eximía al hombre de la necesidad de pensar por sí mismo, la sensología es una nueva forma de poder que prefigura formas de sentir, eximiendo al hombre de sentir por su cuenta, subjetivamente, y sirviéndose de la característica especular de este, dirigiendo los espejos a donde les convenga. Aquí se identifica al hombre con el espejo, con una cosa, no ya con una subjetividad, un sentir interno, sino con un mero objeto que refleja:

Mientras el sentir recayó en el hombre, el nexo entre el mundo orgánico y el mundo inorgánico se regía por afinidades y diferencias. Uno y otro eran concebidos como materia; el primero era una materia sentiente, el segundo no tenía posibilidad de sentir. El hombre, como representaba en el mundo orgánico la forma más compleja y sofisticada de sentir, se arrogaba el derecho a ocupar el sitial más alto en una especie de jerarquía evolutiva de la sensibilidad, que se extendía hasta los animales y las plantas, pero que se detenía ante la materia inanimada. Esta certeza sobre la diferencia específica del hombre respecto a la materia inanimada se ha desmoronado no por un descubrimiento científico que haya identificado la esencia de la vida en un principio distinto al sentir, sino a causa de un proceso histórico-social que exime al individuo de la experiencia del sentir (Perniola 2008, 44-45).

En la ideología, al extrañar el pensamiento, el hombre ya no se distinguía de los animales. En la burocracia, al extrañar el actuar, ya no se distinguía de las plantas. Ahora, la sensología introduce al hombre en el mundo de la cosa, al borrar la distinción entre materia animada, que siente, y materia inanimada, que no siente. El hombre se vuelve así una cosa que siente. Perniola (2006b, 42-43) sostiene que esta es la forma más radical del proceso de alienación que describe Marx y, si bien el remedio de la alienación consiste en la devolución al sujeto de aquello que se le ha extrañado, para Perniola ya no es posible una vuelta a la subjetividad. En su lugar se buscan "remedios homeopáticos" a la alienación, es decir, la respuesta frente al hombre-espejo no consiste en separar al hombre del mundo de las cosas, sino en otra forma de entender al hombre-espejo. 
En efecto, que el hombre pertenezca al mundo de las cosas no es ello mismo problemático, sino que corresponde con el momento histórico-social en el que nos encontramos. Sería una necedad, una ceguera deliberada, querer forzar a la realidad a ser de otra forma en lugar de prestarle atención y aprender a movernos efectivamente en ella. Por eso debemos partir del reconocimiento de que la realidad se nos presenta como enigmática. El enigma, como vimos antes, es profusamente antidualista; en él no cabe pensar en las distinciones orgánico/inorgánico o vital/inerte, sino comprender la unidad de los opuestos y cómo se puede dar un tránsito de lo orgánico a lo inorgánico frente a la misma realidad. Pero esta disolución de distinciones conlleva un compromiso con un materialismo o inmanentismo que Perniola (2006b, 22) ya reconocía en la lectura que Deleuze hace de Leibniz. En ella, se borra la distinción entre el mundo trascendente y el inmanente y acaece una mundanización de la experiencia espiritual.

En El sex appeal de lo inorgánico, Perniola se dedica a profundizar en la experiencia de la sensibilidad del hombre en relación con su ser cosa. En este, señala que Descartes ha subordinado el sentir al pensar, y con ello afirmado una separación radical entre aquello que piensa (siendo sustancia pensante) y aquello que se mueve (sustancia extensa). Sin embargo, "el sentir implica la unión entre espíritu y cuerpo, entre mente y máquina; una cosa pensante puede también no tener cuerpo, pero una cosa sintiente debe tenerlo por fuerza" (Perniola 1998, 15). Nuestro autor señalará que el hombre, al entenderse como cosa que siente, configura el punto de encuentro entre lo orgánico e inorgánico (Bartoloni 2011, 157), donde no puede pensarse su espiritualidad o conciencia como separada de la dimensión corporal. Esta distinción, dirá Perniola $(1998,182)$, solo tiene sentido en un horizonte humanista, mientras que ahora nos encontramos frente a un momento que es post-antropocéntrico, posthumano. La tesis central de este libro referirá a la importancia de pensar una sexualidad neutral, no en función de una subjetividad que busca placer y satisfacción para sí, sino que, al neutralizar la subjetividad, la sexualidad se vuelve neutra, un dar placer entre cosas que sienten, pero no una satisfacción personal de deseo.

Con todo ello, el remedio frente a la sensología y al extrañamiento del sentir consiste en encontrar una forma distinta de sentir; aprender a sentir 
la diferencia y dar un lugar genuino para lo otro. En Del sentir, Perniola (2008, 123-124) indica que esto se alcanza a través de contraponer un sentir activo frente a la pasividad con la que se ha entendido el sentir en nuestra tradición filosófica. Esto supone romper con la identificación que la metafísica ha hecho de la actividad con el pensar y la pasividad con el sentir. La prefiguración del sentir reposa sobre el presupuesto de que otros nos hacen sentir según sus maquinaciones, somos espejos pasivos a la espera de reflejar lo que nos pongan en frente. Sentir activamente supone un obrar sobre nosotros mismos para ser selectivos con aquello que sentimos y para sobrepasar la visión jerárquica que impone la metafísica, en la que el pensar está por encima del sentir. Esto conlleva además una confluencia entre el pensar y el sentir, ya que sentir activamente necesita de un discernimiento, y el pensar adquiere un carácter más pasivo al presentarse como un "recibir lo que llega de fuera, acoger, aceptar lo que se nos presenta como extraño y enigmático" (Perniola 2008, 130). Esta receptividad hacia lo diferente se configura en Enigmas a través del video-zen, la catóptrica barroca, la video-elegancia y el neo-paganismo, mientras que en Del sentir se presenta a través de la aísthēsis y del ménos; el sentir cósmico y el sentir teátrico.

En primer lugar, en el video-zen, al convertirse el hombre en espejo y superar el dualismo del sujeto como sede de la actividad y la conciencia frente a un objeto que es pensado por este, se abole la concepción instrumental de la ciencia que ve en la materia algo a ser explotado por el sujeto (Perniola $2006 b, 44)$. Mientras que la ciencia instrumental se impone sobre lo diferente al apropiárselo, el Zen permite una escucha genuina a la naturaleza. Luego, a través de la novela de Perrault, Perniola muestra la importancia de una disposición amorosa para recibir y acoger lo externo. Orantes, quien reflejaba la realidad tal cual la recibía, por su amor indiscriminado hacia la realidad, se convierte en espejo. En lugar de forzar la proyección de una imagen falsa de Calixta, acepta la realidad tal cual es. La reacción violenta de Calixta puede asemejarse a la violencia del narcisista o del psicótico, quienes no aceptan al otro y buscan afirmarse homólogamente. En cambio, 
la actitud de Orantes puede ser calificada como un amor especular que conlleva la anulación más grande del sujeto que ama: "Éste nada quiere, nada sabe y nada quiere poder, sino que está listo para reflejar con la misma inmediatez tanto a Dios como al demonio" (Perniola 2006b, 47). A diferencia de la incapacidad de tener un diálogo con la pareja al no saber reconocer lo diferente y solo buscar una respuesta de autoafirmación, este tipo de amor, que no busca ni espera nada del amado, sino que está siempre dispuesto a recibir y escuchar, muestra el camino de genuina comprensión que trae consigo el reconocimiento de la diferencia y el abandono del ímpetu de buscar imponer la propia subjetividad a los demás. Finalmente, en Del sentir Perniola explora la aísthēsis (serenidad) y el ménos (posesión) como formas de sentir activas ${ }^{5}$ que permiten una relación con lo otro y hacen frente al nihilismo y neoescepticismo, por un lado, y al fundamentalismo y neofanatismo, por otro.

El primero es un sentir cósmico, un descubrir con gozo la unidad del intelecto con el sentimiento, del alma con el cuerpo, del hombre con el ambiente que lo rodea. El segundo es un sentir teátrico, un ofrecerse con entusiasmo a ser poseídos por fuerzas cuya dinámica resulta enigmática y contradictoria, o, en cualquier caso, extraña a la tranquila identidad del sujeto individual (Perniola 2008, 133).

El primero corresponde a una serenidad frente a la situación enigmática de aceptar que se es parte del universo y el tránsito de lo mismo a lo mismo, sin privilegiar la subjetividad. El segundo, un endiosamiento. Pero ambos son importantes porque entienden de la misma forma el sentir; sentir es dejar marca, huella, como un sello que deja su marca en la cera. Ser como el sello corresponde al sentir cósmico y este necesita de un mundo que lo acoja en el cual pueda dejar su marca. En tal acogimiento y aceptación por parte del mundo "vivimos entonces en plena paz y serenidad y podemos abandonarnos con confianza al abrazo de un cosmos que gobierna nuestro destino"

4 Este amor especular, o amor muerto, como también lo llama Perniola, podría mostrarse en relación con la idea de una sexualidad neutral, mencionada previamente, ya que en ella se borra la idea de un sujeto que busca satisfacción o placer y solo se entrega como cosa que siente. Sin embargo, tal afirmación requeriría de mayor precisión y no es el objeto del trabajo presente.

5 Esta última también está presente en Enigmas, como parte del apartado sobre el hacerse cosa neo-pagano. 
(Perniola 2008, 135). Por su parte, el sentir teátrico es más como ser la cera que toma la forma de quien imprime su sello en ella, es ser una vestidura o "túnica de piel que reclama ser habitada" (Perniola 2008, 135), poseída por fuerzas extrañas y grandiosas. La posesión está fuertemente asociada al ritual, por el aura que este trae. El entusiasmarse refiere a ser habitado por la divinidad; sin embargo, más allá de la creencia metafísica en dioses o espíritus, lo que se constituye es una forma de sentir que genera "asombro por algo que está, de forma inesperada e incluso contra toda verosimilitud, presente aquí y ahora, en carne y hueso" (Perniola 2008, 136), sentir que algo más allá de lo creíble o cotidiano está presente en este instante. Por ejemplo, en la posesión no-partícipe, apolínea, que describe Perniola, se da un momento de tensión entre opuestos, una experiencia contradictoria que escapa radicalmente a nuestro mundo humano, que a nuestra razón mundana parece inconcebible. Sin embargo, se da realmente, y asombra por su inconmensurable diferencia y su astucia.

\section{§3. Lo posthumano y el materialismo vitalista de Braidotti}

Rosi Braidotti es una pensadora vinculada al nuevo materialismo, una etiqueta que refiere a diversos autores interesados por pensar la realidad como una unión entre materia y espíritu, y abolir los dualismos presentes en la metafísica tradicional. Esta línea de trabajo se origina en el ámbito de los estudios feministas y va a la par con el cuestionamiento sobre la diferencia, subrayando, además, un ímpetu por pensar la realidad en su complejidad y en su aspecto material y concreto, evitando apelar a ideales ordenadores del mundo. Entre las autoras y autores del nuevo materialismo también están Donna Haraway, Jane Bennett y Manuel DeLanda.

La problemática del posthumanismo, objeto de discusión del libro de Braidotti, Lo posthumano, consiste en una reflexión y crítica sobre los presupuestos del pensamiento y la tradición humanista. Braidotti parte de la consideración de que la época actual es profundamente posthumana y reclama que la consideremos como tal. La tradición humanista, por su parte, ha privilegiado la cualidad de la racionalidad por encima de la sensibilidad y, en base a su definición de hombre como animal racional, 
ha justificado órdenes jerárquicos en los que el hombre está siempre en la cima de la cadena, superado (en algunos casos) únicamente por Dios. Además de ello, esta tradición ha pensado la idea de hombre de una forma parcial y limitada, a saber, varón, blanco, heterosexual, urbanizado, hablante de una lengua estándar (Braidotti 2015, 82). De esta manera, apelando al ideal de racionalidad, se ha otorgado a quienes participan más del ideal civilizatorio un grado mayor de "humanidad" frente a quienes discrepan del ideal hegemónico y quedan relegados a ser subalternos o vistos como más próximos a las bestias. Esta discriminación se basa en la incapacidad de la metafísica de la presencia de pensar detenidamente la diferencia, creando más bien identidades fijas en una situación donde unas se imponen sobre otras (González 2018, 174). Por ello, resulta de suma importancia pensar la noción de diferencia y superar el horizonte antropocéntrico y humanista. Aunque en la situación actual se esté dando un postantropocentrismo, gracias a los progresos científicos y el capitalismo biogenético (pensando al hombre como materia bioquímica), no por ello este postantropocentrismo es posthumano (Braidotti 2015, 82). Este último conlleva un desplazamiento de los intereses del hombre y de su afán de poder y control sobre el mundo para entrar en una relación horizontal de sostenibilidad con el mundo que le rodea y del cual es co-dependiente. Para ello será importante abrir paso a la consideración de la diferencia; no solo distintas formas de vida, sino distintas configuraciones de la subjetividad que manifiesten diversas formas de ser que potencien la heterogeneidad de alternativas a la hegemónica. Así es como el problema del posthumanismo tiene para Braidotti simultáneamente una dimensión ética y ontológica (González 2018, 176). Esto, en lugar de una actitud meramente negativa del pensamiento de la diferencia, constituye su dimensión positiva o afirmativa (González 2018, 174-175; Sargento 2013, 115-116).

Braidotti sostiene su pensamiento posthumanista en una concepción ontológica de la realidad que llama materialismo vitalista. A diferencia del dualismo cartesiano entre sustancia pensante (mente) y sustancia extensa (cuerpo), el materialismo vitalista toma inspiración spinozista, rechazando la distinción dualista y definiendo a "la materia como vital y capaz de autoorganización (...). Desde el momento en que esta aproximación rechaza cualquier tipo de trascendentalismo, es conocida también como 
'inmanencia radical'"' (Braidotti 2015, 72). En otras palabras, hay una unidad en la materia, de forma que la "vitalidad" de los organismos no proviene de una facultad racional externa a la naturaleza de la materia y radicalmente distinta de esta. En su lugar, la materia es capaz de auto-organizarse, es decir, configura diversas formas de vida de-abajo-hacia-arriba sin necesidad de un principio de organización teleológico, racional y externo. La materia misma posee una capacidad inteligente de organizarse a partir de su relación con el medio ambiente. Esto, en consecuencia, transformará la noción de sujeto de algo abstracto, individual y aislado, a una noción relacional, moldeable y en constante contacto con lo que le rodea (Braidotti $2015,76)$. Sargento $(2013,116)$ llama a esta configuración de subjetividad una "subjetividad neutralizada" puesto que el sujeto (entendido en términos modernos) se retira como el principio organizativo y supervisor, dando paso, más bien, a la "otredad" de la materia. La fuente de esta capacidad autoorganizadora que es la fuente generadora de vida se llamará zoe:

El postantropocentrismo está marcado por el surgimiento de la política de la vida (Rose, 2008). La vida, en vez de ser definida como propiedad exclusiva y derecho inalienable de una sola especie, la humana, sobre todas las demás, en vez de ser santificada como una tesis predeterminada, es entendida como proceso interactivo y sin conclusiones. Esta aproximación vitalista a la materia elimina los confines binarios entre aquella parte de la vida, sea orgánica, sea discursiva, tradicionalmente reservada al anthropos, es decir, el bios, y la parte más amplia de la vida animal y no-humana, también conocida como zoe. Zoe como fuerza dinámica de la vida en sí, capaz de autoorganización, permite la vitalidad generativa (Braidotti, 2008, 2011). Zoe es la fuerza transversal que corta y vuelve a zurcir especies, dominios y categorías precedentemente separadas. El igualitarismo zoe-centrado es, para mí, el núcleo de la inflexión postantropocéntrica: es una respuesta materialista, laica, fundada y concreta a la oportunista mercantilización transespecie que es la lógica del capitalismo avanzado (Braidotti 2015, 77).En efecto, zoe es entendida como la vitalidad común que subyace a todas las formas de vida y de la que surgen todas estas. A diferencia de bios, que define la vida como prerrogativa del hombre, en tanto conciencia e inteligencia, zoe es entendida como la fuerza generativa que impulsa a la materia a configurarse y reconfigurarse de distintos modos, de forma que la subjetividad no es algo cerrado sino en constante movimiento, cambio e interacción con otras subjetividades y con el ambiente. Al desterrar el principio trascendente de la vida, ninguna forma de vida o configuración de subjetividad está por encima de las demás, sino que todas están al mismo nivel, en interrelación dinámica, proviniendo de la misma fuente. Así, se transforma la 
relación de distintas formas de vida y subjetividades de una jerárquica a una de horizontalidad e igualitarismo entre las distintas configuraciones de zoe. Esta nueva forma de relación igualitaria prioriza el respeto mutuo y la humildad, en lugar de la apropiación y explotación presente en la lógica capitalista. La política de la vida pone a esta última como un valor intrínseco por ser vital, que debe ser resguardado y respetado, porque son manifestaciones de zoe, de la que somos parte. González (2018, 180; cursivas añadidas) señala que "[c]on la noción de zoé, de vital importancia en su ontología, Braidotti refiere a la "vida misma", la cual es entendida como potencia o energía vital productiva y creativa". Esta energía vital y creativa es la que otorga el elemento vitalista al materialismo de Braido$\mathrm{tti}$, pues denota una valoración de la vitalidad como potencia creativa, expresión de vida, sin más.

Como he mencionado antes, el sujeto se configura en relación con su entorno y está en una constante y dinámica interacción con otras subjetividades. Esta interconexión del sujeto significa que para comprenderse debe mirarse a sí mismo y a la malla de relaciones en que se encuentra, adquiriendo conciencia de las formas en las que afecta y es afectado (González 2018, 183). Cabe resaltar que este reconocimiento de la interrelación debe tomar la forma de una conciencia ecológica, un "devenir imperceptible"6 que lleve a una renuncia ascética de la identidad propia para configurar una apertura y sensibilidad que permitan una suerte de "impersonalidad ascética”, el encuentro con la diferencia (González 2018, 187). Así, “[e]l concepto de codependencia sustituye aquí al de reconocimiento, como la ética de la sostenibilidad sustituye a la filosofía moral de los derechos" (Braidotti $2015,112)$, pues no se trata de otorgar derechos en función de un estatus como "humano", sino de buscar relaciones sostenibles y empáticas con lo que nos rodea puesto que, aunque diferentes, somos parte de lo mismo y somos co-dependientes. Respetar las distintas configuraciones de zoe porque todas son vida, a un mismo nivel relacional, horizontal, sin jerarquías ni privilegios. De hecho, la noción de subjetividad que se configura aquí

6 Gonzales $(2018,187)$ señala que este es un término que Braidotti retoma de Deleuze. El devenir-imperceptible es entendido como un sentido cósmico de unión con la naturaleza mediante el cual permitimos que la materia vital (zoe) se exprese a través de nosotros (Cfr. Braidotti 2006, 173). Por ello, el devenir-imperceptible supone un abandono de nuestro aferro a la identidad propia como sujeto individual que se impone y abre paso en el mundo. En su lugar, al devenir-imperceptible, somos conscientes de una responsabilidad vinculada a una sensación de interconectividad que supone una ética empática y afectiva que atraviesa a las distintas especies, el espacio y el tiempo (Braidotti 2006, 262). 
difiere radicalmente y es más amplia y ambigua que la del sujeto moderno. Sin embargo, debemos ser cuidadosos, ya que, aunque no haya limites estables y definidos que determinen la identidad de los individuos, esto tampoco debe tomarse como una mezcla total en una unidad indiferenciada: "Sé que esto puede parecer inconsciente y aventurado, pero yo me quedo de este lado: del lado de lo que ya no se identifica con las categorías dominantes del sujeto, pero que no es aún del todo libre de las jaulas de la identidad, o sea, del lado de lo que es diferente y sigue diferenciándose de sí y, por ende, está cerca de zoe, el sujeto postantropocéntrico" (Braidotti $2015,98)$. El sujeto postantropocéntrico es permeable, pero justamente por eso, retiene cierta forma, con ciertas barreras, difusas pero existentes. No se trata aquí de un devenir todo con todo, sino de la posibilidad de una afectación mutua que permita un cambio gradual; nos entendemos como seres diferentes, pero parte de lo mismo.

Tanto la permeabilidad del sujeto con su entorno como la disolución de la distinción mente/cuerpo, y el consiguiente rechazo de la identificación del sujeto con la conciencia o el pensar, implican la necesidad de una revalorización sobre el rol que ocupa la sensibilidad y afectividad en la conformación de la subjetividad. Esto nos dirige, en primer lugar, a pensar en la noción de subjetividad como necesariamente encarnada, perteneciente a un cuerpo, y este, a su vez, situado en un contexto particular, histórico y geográfico. Ser sujeto es, para Braidotti, ser cuerpo (González 2018, 180). En segundo lugar, entendiendo a la subjetividad como encarnada, las pasiones, emociones y los sentimientos "pasan a ocupar un lugar de privilegio en el estudio del modo con que el sujeto construye tanto la imagen de sí mismo como del mundo que le rodea, completando y complementando a la racionalidad, la facultad que la filosofía tradicional había considerado como la más propia y específicamente humana" (González 2018, 181). Se vuelve apremiante prestar atención a nuestra sensibilidad, no como algo que debe ser controlado por y subordinado a la razón, sino como una facultad inmensamente relevante para entender quiénes somos.

Ahora bien, dentro de su planteamiento, Braidotti explora tres sentidos del devenir al que el sujeto nómade o posthumano se enfrenta y detalla los tipos de interrelación que se instauran entre subjetividades en esos 
devenires. Estos son: devenir animal, devenir tierra y devenir máquina. El primero refiere a la solidaridad transespecie que surge de una perspectiva horizontal y no jerárquica y comprende el ver a un hombre como animal entre animales. El segundo, partiendo de la perspectiva zoecéntrica y de la co-dependencia, exhorta a una preocupación por el medioambiente, el ecosistema y la sostenibilidad de este, del que dependen las diversas formas de vida. En esta línea, y de acuerdo con la cuestión de la diferencia, no se trata aquí de un humanismo compensatorio que trate de "humanizar" a las distintas formas de vida y compartirles nuestros derechos humanos, ni tampoco de pretender salvar al medio ambiente meramente porque es la condición de posibilidad de la subsistencia de nuestra especie. Por el contrario, supone reconocer al otro en su diferencia, en la alteridad con uno, y que en esa diferencia aun sea admitido, respetado e incluido dentro del mundo de la vida. Así mismo, no debemos cuidar el medio ambiente por un temor a nuestra extinción, sino por respeto a la zoe y humildad frente a sus distintas manifestaciones, entendiendo que no somos dueños de la naturaleza para explotarla a voluntad.

Por último, el devenir máquina explora la relación entre la naturaleza y la cultura, lo natural y lo artificial y, a través de la permeabilidad del sujeto, pone en cuestión la distinción radical entre estos, encontrando más bien un continuo relacional. Los avances en biogenética, robótica e informática, entre otras ciencias, han tenido por consecuencia la difuminación de las barreras entre lo animado y lo animado, configurando una nueva forma de entidad, distinta ya del mero humano, que, siguiendo a Donna Haraway en El manifiesto cyborg, llamamos cyborg (González 2018, 189). Esta tercera dimensión, sin duda la más nueva y radical, es crucial para poner en cuestión la forma en la que se configura la subjetividad y mostrar el modo en que el cuerpo y su interacción con el medio ambiente juegan un rol determinante en dicha configuración. Al considerar que no hay una separación categórica entre materia orgánica e inorgánica y que se pueden configurar nuevas formas de vida, con distintas percepciones y experiencias del mundo a partir de distintas aglomeraciones de materia autoorganizada según una potencia vital (zoe), es posible la crítica al monopolio de la vida por parte del hombre. 


\section{$\S 4$. Discusión y conclusiones}

Luego de haber expuesto algunas de las ideas centrales de ambos autores, quisiera dedicar esta última sección a una breve comparación de ambas posturas para mostrar y resaltar ciertas afinidades entre ellas, así como marcar ciertas diferencias. Con ello, no pretendo ser rigurosamente exhaustivo, sino meramente mostrar, a grandes rasgos, las potenciales convergencias y divergencias entre dos autores que muestran cierta afinidad por una visión materialista e inmanente de la realidad.

En primer lugar, ambos autores coinciden en tomar una postura crítica frente a los presupuestos de la metafísica occidental tradicional. Esta, habiendo privilegiado la identidad, habría buscado fijar la realidad en conceptos y certezas, cayendo así en esencialismos que, privilegiando la identidad simplificaban la complejidad de la realidad, y que constituyen un daño y una agresividad contra lo otro. Pensar debidamente la realidad significa, para ambos, prestar atención a la cuestión de la diferencia. Sin embargo, cada autor enfatiza matices distintos de la noción de diferencia. Para Perniola, la diferencia es tomada como una alteridad radical, sobre todo en los casos de posesión, aunque también admite una diferencia más sutil en Contra la comunicación al enfatizar la importancia de reconocer al interlocutor como otro y entablar un conflicto real con él. Braidotti, en cambio, acentúa el aspecto afirmativo o positivo de la diferencia que busca ser inclusivo y diverso. Además, el rol que cumple la diferencia es distinto. Para Perniola la diferencia es una forma de hacerle frente al caos comunicativo (recalcando la importancia del orden simbólico y la mediación) y al extrañamiento del sentir (buscando formas de sentir que hagan frente a la impersonalidad sin plantear un retorno a la subjetividad), mientras que para Braidotti se trata de respetar a las diferentes y diversas formas de vida y cultivar con humildad nuestra condición humana.

Cabe mencionar, además, que ambos reconocen en el poder económico contemporáneo una fuente central de la problemática o el predicamento en el que nos encontramos. En el caso de Perniola, la prefiguración del sentir permite que se negocie con el sentir colectivo como moneda de intercambio, para el beneficio de unos particulares dentro de la lógica del 
mercado capitalista. Braidotti también critica la lógica capitalista, pero desde el ángulo de la explotación irrestricta de la naturaleza para la ganancia máxima de intereses privados, que es devastadora para la vida. Por otra parte, aunque ambos reconozcan que la situación actual es posthumana, Perniola, como vimos, explicita que no considera que esto se deba a un descubrimiento científico sobre "el secreto de la vida", sino a una condición histórico-social en la que la forma en que entendemos el mundo se ha transformado y el sentir ha sido extrañado del sujeto. Braidotti también reconoce la importancia del contexto y el presente histórico como posthumanos, pero sí aventura explicaciones sobre cómo la biogenética y los avances tecnológicos han influenciado en el desarrollo de esta nueva concepción de la realidad.

Asimismo, ambos adoptan una postura materialista e inmanente que es profusamente antidualista, que rechaza los dualismos mente/cuerpo, animado/inanimado, natural/artificial, y la actitud simplista y maniquea, optando más bien por confrontar la complejidad de la realidad. Esto concuerda, además, con que ambos autores le den un rol esencial y prioritario a la sensibilidad para entender al sujeto en el contexto contemporáneo. Sin embargo, más que discutir las implicancias metafísicas de su propia posición, a Perniola le importa el materialismo porque reconoce en él una herramienta útil para dar sentido a la realidad contemporánea (Hanza 2017, 58-59). En cambio, Braidotti tiene un interés explícitamente ontológico al poner en cuestión a la metafísica de la presencia, pues busca mostrar cómo es posible la configuración de diversas formas de vida, así como cambiar radicalmente nuestra noción de sujeto.

En este punto es importante reparar en un posible conflicto entre el materialismo de Braidotti y la perspectiva enigmática de Perniola. Braidotti llama a su perspectiva un "materialismo vitalista" $y$, frente a ello, cabe preguntarse: ¿en qué sentido utiliza la palabra "vitalista"?, ¿podría ser objeto de la crítica que Perniola hace al vitalismo? Recordemos que para Perniola, el problema del vitalismo consistía en que, al exhortar la vida por la vida misma, acepta indiscriminadamente cualquier manifestación de creatividad vital, sin distinguir a las muestras de ingenio de las tonterías. El problema para Perniola no radicaría meramente en la apelación al mundo de la vida y de 
la experiencia vital, sino en que esta se emplee como manera de evitar el conflicto, señalando que la realidad es irracional y contradictoria y que no hay más que hacer. El vitalismo tendría, así, afinidad con el nihilismo antes que con una postura crítica para discernir sobre lo que tiene sentido.

Ahora bien, es innegable que Braidotti considera la vida misma como un valor, y que la diversidad de manifestaciones vitales y de la potencia creativa de estas es deseable por sí misma como acogimiento de la diferencia. Sin embargo, considero que de todas formas hay una diferencia sustancial entre el vitalismo que critica Perniola y el sentido que le da Braidotti, pues la autora no pretende evitar la experiencia del conflicto o de la diferencia, ni utiliza el concepto para exaltar la creatividad humana como ser con vitalidad a diferencia de la naturaleza, sino que el concepto de materialismo vitalista sirve justamente para criticar la distinción entre ser humano vital y el resto de la naturaleza inanimada, mostrando que basta observar el mundo de lo inanimado para entender cómo se forma la conciencia y cómo la distinción animado/inanimado pierde su sentido y, por lo tanto, todo tiene fuerza vital. Más aún, mientras que el vitalismo comunicativo busca la afirmación de la vida sobre la muerte, permanencia e inmortalidad, para la zoe, que es potencia vital, no hay distinción entre la vida y muerte de los individuos, sino un reconocimiento de su condición efímera, pasajera, y una aceptación del devenir otra cosa. No hay un impulso de aferrarse a la vida a como dé lugar, sino un aprecio por el momento finito que se llega a experimentar como sujeto, para que, con la muerte, la materia sea reconfigurada a través de la zoe como otra cosa, dejando de lado el egocentrismo.

Por último, si bien el vitalismo que critica Perniola se asemeja al nihilismo al disolver el sentido y las diferencias, no es precisamente Braidotti quien propugne tal disolución. Ella, al igual que Perniola, considera que el nihilismo es una cuestión que debe ser combatida, mostrando las formas en que se puede pensar el devenir (o el tránsito) al tomar en serio las diferencias. Que el sujeto sea nómade y permeable no niega que es algo que está siendo de una determinada manera en un determinado momento, y que ciertas cosas valen para ese sujeto y otras no en ese momento determinado. Asimismo, aunque Braidotti tenga una noción relacional de subjetividad, enfatiza que eso no debe disolver las diferencias jamás; que "somos parte de lo mismo, 
pero no somos uno y lo mismo" (todos somos manifestaciones de zoe pero somos particulares, individuales, múltiples y diversos). La noción de enigma de Perniola comparte algo de esto, en tanto ella también supone que hay un fundamento común que garantiza la comunicabilidad y el tránsito de lo mismo a lo mismo (es decir, de distintas manifestaciones, diferentes, de esa misma fuente común que es la realidad).

Para concluir, aunque podría entrarse en mayor detalle sobre cada uno de estos puntos, me limito a señalar las conexiones presentes. Si bien, en buena medida, podemos decir que ambos toman posiciones afines con respecto al sentir y a la necesidad de pensar la subjetividad de forma radicalmente distinta a como se ha concebido tradicionalmente, encontramos que los problemas a los que se enfrentan ambos autores están enfocados de forma distinta. No podría decirse, sin más, que Braidotti explora las ramificaciones ontológicas de un pensamiento como el de Perniola, ya que hay diferencias sustanciales, sobre todo con respecto al vitalismo. No obstante, no por ello se pierde de vista que ambos están profundamente interesados por pensar en cómo debemos vivir en el mundo contemporáneo. No debería suponerse una búsqueda de "quién responde más acertadamente a la situación contemporánea" porque las situaciones a las que responden, aunque compartan muchos aspectos, tienen matices distintos. Mientras que Perniola está más preocupado por plantear una forma de sentir activo que permita orientarnos y evitar que nos dejemos llevar por la sensología y la comunicación, Braidotti se enfoca en una conciencia planetaria, o cósmica, que genere un sentido de responsabilidad con lo diferente. Lo que sí podemos preguntarnos es si ambas posturas son incompatibles, y la respuesta, a mi parecer, es un contundente no. Ambos buscan una forma de orientarnos en el mundo que sea sostenible y sensata, y que se enfrente al nihilismo, mostrando cómo es que podemos encontrar sentido y valor dentro del tránsito y del devenir, apelando al mundo inmanentemente y eliminando la distinción entre sujeto orgánico y materia inorgánica. 


\section{Bibliografía}

Fuentes citadas:

Bartoloni, Paolo, 2011. Thinking Thingness: Agamben and Perniola. Annali D'Italianistica 29, 141-162. http://www.jstor.org/stable/24016418. Consultado 14 noviembre 2019.

Braidotti, Rosi, 2006. Transpositions. On Nomadic Ethics. Cambridge: Polity Press.

- 2015. Lo posthumano. Traducción de Juan Carlos Gentile Vitale. Barcelona: Gedisa.

González, Brais, 2018. La ética diferencial de Rosi Braidotti. AGORA 37 (2), 173-191. https://doi.org/10.15304/ag.37.2.4364. Consultado 14 noviembre 2019.

Hanza, Kathia, 2017. Del sentir impersonal. Los estudios de Mario Perniola sobre la era estética. Revista del Instituto Riva-Agüero 2 (1), 57-80. https://doi.org/10.18800/ revistaira.201701.002. Consultado 14 noviembre 2019.

Perniola, Mario, 1998. El sex appeal de lo inorgánico. Traducción de Mario Merlino. Madrid:Trama.

- 2006a. Contra la comunicación. Traducción de Carlo R. Molinari Marotto. Buenos Aires: Amorrotu.

- 2006b. Enigmas: Egipcio, barroco y neo-barroco en la sociedad y el arte. Traducción de Javier García Melenchón. Murcia: Cendeac.

- 2008. Del sentir. Traducción de César Palma. Valencia: Pre-Textos.

Sargento, Pedro, 2013. New Materialism and Neutralized Subjectivity. A Cultural Renewal? Cultura. International Journal of Philosophy of Culture and Axiology 10 (2), 113-125. https://doi.org/10.5840/cultura201310216. Consultado 14 noviembre 2019.

Fuentes consultadas:

Marino, Patricia, 2010. Review of Mario Perniola, 'The Sex Appeal of the Inorganic' (Massimo Verdicchio, translator). Journal of the History of Sexuality 19 (1), 179182. https://www.jstor.org/stable/40663378. Consultado 14 noviembre 2019. Perniola, Mario, 2010. El sex appeal del bisturí. Metapolítica 12 (69), 24. http:// ezproxybib.pucp.edu.pe:2048/login?url=http://search.ebscohost.com/login. aspx?direct=true \&db=fua\&AN=53148586\&lang=es\&site=eds-live\&scope=site. Consultado 14 noviembre 2019.

Perniola, Mario y Deborah Amberson, 2005. Remembering Derrida. SubStance 34 (1), 48-49. https://www.jstor.org/stable/3685615. Consultado 14 noviembre 2019. 